\title{
Erratum to: Use of parenteral colistin for the treatment of multiresistant Gram-negative organisms in major burn patients in South Korea
}

\section{Y. S. Cho $\cdot$ H. Yim $\cdot$ H. T. Yang $\cdot$ J. Hur $\cdot$}

W. Chun $\cdot$ J. H. Kim $\cdot$ B. C. Lee $\cdot$ D. K. Seo $\cdot$

J. M. Park $\cdot$ D. Kim

Published online: 11 October 2011

(C) Springer-Verlag 2011

\section{Erratum to: Infection}

DOI 10.1007/s15010-011-0192-7

In the original published article, unfortunately co-author J.M. Park was omitted from the authorship.

The online version of the original article can be found under doi:10.1007/s15010-011-0192-7.

Y. S. Cho · H. Yim · H. T. Yang - J. Hur · W. Chun ·

J. H. Kim · J. M. Park · D. Kim (凹)

Department of Surgery, Burn Center,

Hangang Sacred Heart Hospital,

Hallym University Medical Center,

94-200 Youngdeungpo-dong, Youngdeungpo-gu,

Seoul 150-719, Korea

e-mail: dohern@hallym.ac.kr

Y. S. Cho

e-mail: maruchigs@hallym.or.kr

H. Yim

e-mail: pkgom@hallym.or.kr

H. T. Yang

e-mail: yht0227@hanmail.net

J. Hur

e-mail: hammerj@hallym.or.kr

W. Chun

e-mail: chun0414@hallym.ac.kr

J. H. Kim

e-mail: mcjemba@hanmail.net
B. C. Lee

Department of Psychiatry, Burn Center,

Hangang Sacred Heart Hospital,

Hallym University Medical Center,

Youngdeungpo-gu, Seoul, Korea

e-mail: leeboungchul@hallym.or.kr

D. K. Seo

Department of Plastic Surgery,

Burn Center, Hangang Sacred Heart Hospital,

Hallym University Medical Center,

Youngdeungpo-gu, Seoul, Korea

e-mail: sdkps@hallym.or.kr

\section{J. M. Park}

Department of Dermatology, Haeundae Paik Hospital,

College of Medicine, Inje University, Busan, Korea

e-mail: jinmo@paik.ac.kr 